\title{
Communities unfolding in multislice networks
}

\author{
V. Carchiolo, A. Longheu, M. Malgeri, and G. Mangioni
}

1 Vincenza Carchiolo. Dipartimento di Ingegneria Informatica e delle Telecomunicazioni, University of Catania, ITALY. E-mail: car@ diit.unict.it

2 Alessandro Longheu. Dipartimento di Ingegneria Informatica e delle Telecomunicazioni, University of Catania, ITALY. E-mail: alongheu@ diit.unict.it

3 Michele Malgeri. Dipartimento di Ingegneria Informatica e delle Telecomunicazioni, University of Catania, ITALY. E-mail: mm@diit.unict.it

4 Giuseppe Mangioni. Dipartimento di Ingegneria Informatica e delle Telecomunicazioni, University of Catania, ITALY. E-mail: gmangioni@ diit.unict.it

Summary. Discovering communities in complex networks helps to understand the behaviour of the network. Some works in this promising research area exist, but communities uncovering in time-dependent and/or multiplex networks has not deeply investigated yet. In this paper, we propose a communities detection approach for multislice networks based on modularity optimization. We first present a method to reduce the network size that still preserves modularity. Then we introduce an algorithm that approximates modularity optimization (as usually adopted) for multislice networks, thus finding communities. The network size reduction allows us to maintain acceptable performances without affecting the effectiveness of the proposed approach.

\section{Introduction}

Communities structure detection in complex networks is a research field that gained a considerable attention in the last few years. Such interest is due to the possibility to discover hidden behaviours by simply studying the network partitioning into communities. Several methods to address the problem of community uncovering (see [7] for an overview) can be found in literature. However, few of them consider the more general case of communities in time-dependent networks ([5][9][16][2][6]) and/or multiplex networks. On the other hand, networks whose topology evolves over time are quite common ([10][13]). In this case, studying the community structure by simply considering the network obtained by adding together all of its snapshots over time can be too simplistic, and it would not permit to investigate about the temporal evolution of communities. To address this problem, recently Mucha et al. [14] presented a general framework to study the community structure of arbitrary multislice networks, i.e. a set of individual networks linked together by the use of inter-slice links. Multislice networks are general enough to be used to model time-varying, multiplex and multiscale networks. To assess the quality of a given partition into communities, Mucha et al. [14] extended the modularity function, originally introduced in $[15]\left(Q_{N G}\right)$, to be applied to the more general case of multislice networks $\left(Q_{\text {multislice }}\right)$. 
A natural way to explore communities structure in multislice networks is by direct optimization of the $Q_{\text {multislice }}$ function. Unfortunately, the exact optimization of the $Q_{N G}$ modularity function is an $N P$-complete problem [4], and the optimization of the $Q_{\text {multislice }}$ function presents a similar problem. To deal with this problem, several approximation methods have been developed (see [7] for an overview). Among them, the Louvain method devised by Blondel et al. [3] is one of the fastest yet sufficiently accurate algorithm.

In this work we present an algorithm inspired by [3] to discover communities in large multislice networks. The paper is organized as follows. In section 2 we introduce multislice networks discussing about previous works in this topic. Section 3 presents a method to reduce the size of a multislice network while preserving modularity. Section 4 illustrates our algorithm to discover community structure in multislice networks. Finally, in section 5 conclusions and future works are discussed.

\section{Communities in multislice networks}

Real networks often are inherently dynamic, i.e. they change over time. Community structure in such networks cannot be effectively analyzed neither only considering a single time snapshot nor studying a new network obtained by a sort of "sum" of all the variations across time. On the other hand, traditional approaches to community discovering are not generally well suitable to manage multiplex (or multi-layer) networks, where multiple edges between couple of nodes are allowed. Multiplex networks model different kind of relations between nodes and can be, alternatively, represented as a superimposition of distinct layers, each of which being the network obtained by considering a single relation.

To address these issues, in [14] the authors proposed a framework to study community structure in multislice networks. A multislice is a network composed by a set of network slices linked together by inter-slices links. As an example of such a network, in figure 1 it is reported a network composed by three slices coupled each other by a set of links depicted using dotted lines. Multislice networks can be used in many contexts. For instance, a multiplex network can be simply represented by a multislice network by mapping each layer of the network to a slice. Moreover, a time varying network can be mapped to a multislice network where each slice is a single instant snapshot network.

In [14] the authors also propose a multislice extension of the Newman's modularity function, thus providing a metric to assess the quality of a given partition into communities of a multislice network.

In particular, given a multislice network, the multislice generalization of modularity for unipartite, undirected network slices and couplings is:

$$
Q_{\text {multislice }}=\frac{1}{2 \mu} \sum_{i j s r}\left\{\left(A_{i j s}-\gamma_{s} \frac{k_{i s} k_{j s}}{2 m_{s}}\right) \delta_{s r}+\delta_{i j} C_{j s r}\right\} \delta\left(g_{i s}, g_{j r}\right)
$$

Where $i$ and $j$ range over all nodes, $s$ and $r$ range over all slices, $A_{i j s}$ is the element of the adjacency matrix of the slice $s$ (intra-slice), $C_{j s}$ is the link between node $j$ in slice $s$ and node $j$ in slice $r$ (inter-slice coupling), $k_{i s}\left(k_{j s}\right)$ is the degree of node $i(j)$ in slice $s, m_{s}$ is the number of links in slice $s, \gamma_{s}$ is a resolution parameter and $\mu$ is a normalization factor.

Equation 1 can be considered as composed by two terms, the first one takes into account the contribution to the modularity given by each slice (it looks like Newman's modularity), whereas the second term is the contribution given by the inter-slices coupling. 


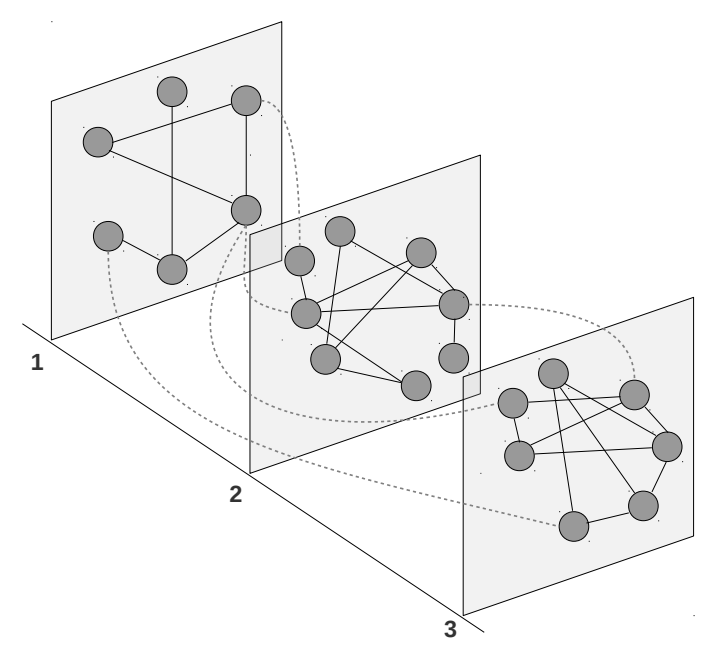

Fig. 1. An example of a three slices network

The modularity function in equation 1 plays a double role: 1) it is used to assess the quality of a given partition and 2) it can be exploited to discover community structure by direct optimization. Unfortunately, as discussed in section 1, the exact modularity optimization is presumably an $N P$-complete problem (similarly to what has already been observed for Newman's modularity in [4]). To overcome this computability matter, in this paper we propose a greedy method to optimize $Q_{\text {multislice }}$ inspired by the Louvain algorithm [3]. In particular, our algorithm makes extensively use of a network size reduction method that we have specifically devised for multislice network, explained in the next section.

\section{Size reduction in multislice networks}

Reducing the size of multislice networks is useful to implement greedy optimization for $Q_{\text {multislice }}$. To achieve this, let $G_{m}$ a multislice network with undirected network slices and coupling (this does not affect generality).

Note that, by definition, each node in slice $s$ is connected only with the same node in slice $r$, that is $C_{i j s r}=0 \forall i \neq j$, then $C_{j s r} \equiv C_{i j s r} \forall i, j$. This equivalence implies that the term $\delta_{i j} C_{j s r}$ in equation 1 can be substituted by the equivalent $C_{i j s r}$, so resulting equation is as follows:

$$
Q_{\text {multislice }}^{*}=\frac{1}{2 \mu} \sum_{i j s r}\left\{\left(A_{i j s}-\gamma_{s} \frac{k_{i s} k_{j s}}{2 m_{s}}\right) \delta_{s r}+C_{i j s r}\right\} \delta\left(g_{i s}, g_{j r}\right)
$$

Where $\delta_{i j}$ has been included into the coupling term.

By definition, for every partition into communities of $G_{m} \Rightarrow Q_{\text {multislice }}^{*} \equiv Q_{\text {multislice }}$. 
Now let $\operatorname{Com}_{s}:\{1, \ldots, N\} \rightarrow\left\{1, . . M_{s}\right\}$ be a partition of slice $s$ of the network into $M_{s}$ communities. The function $\operatorname{Com}_{s}$ assigns a community index $\operatorname{Com}_{s}(i)$ to node $i$ in slice $s$ of the network $G_{m}$. Let us consider the reduced network $G_{m}^{\prime}$ obtained as in the following:

- In every slice $s$ we replaced each community with a single node.

- The intra-slice weight $w_{m n s}^{\prime}$ between the nodes $m$ and $n$ of slice $s$ of the reduced network $G_{m}^{\prime}$ is defined as in the following:

$$
w_{m n s}^{\prime}=\sum_{i} \sum_{j} A_{i j s} \delta\left(\operatorname{Com}_{s}(i), m\right) \delta\left(\operatorname{Com}_{S}(j), n\right) m, n \in 1, \ldots, M_{s}
$$

i.e. $w_{m n s}^{\prime}$ is the sum of all the links connecting vertices in the corresponding communities.

- The inter-slice weight $C_{m n s}^{\prime}$ between node $m$ in slice $s$ and node $n$ in slice $r$ of the reduced network $G_{m}^{\prime}$ is defined as in the following:

$$
C_{m n s r}^{\prime}=\sum_{i} \sum_{j} C_{i j s r} \delta\left(\operatorname{Com}_{s}(i), m\right) \delta\left(\operatorname{Com}_{r}(j), n\right) m \in 1, \ldots, M_{s}, n \in 1, \ldots, M_{r}
$$

i.e. $C_{m n s r}^{\prime}$ is the sum of all the links connecting vertices in community $m$ in slice $s$ with vertices in community $n$ in slice $r$.

In other words, the reduced multislice network is obtained by collapsing each community in one node and by properly setting the weights of both inter-slice and intra-slice links.

Figure 3 presents an example of the application of the proposed size reduction method . Figure 2(a) shows the original multislice network $G_{m}$ composed by three slices where nodes belonging to a community are depicted using the same colour. Figure 2(b) shows the reduced multislice network $G_{m}^{\prime}$ composed (as the original one) of three slices, where each community has been replaced by one node and link weights are recomputed by using equations 3 and 4 .

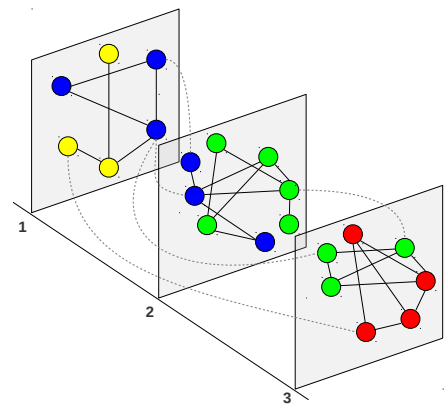

(a) Original multislice network - $G_{m}$

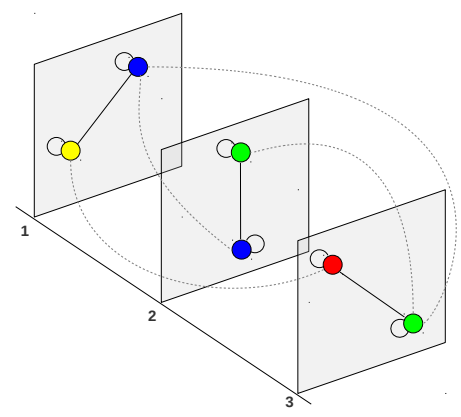

(b) Reduced multislice network - $G_{m}^{\prime}$

Now we want to prove that the $Q_{\text {multislice }}^{*}$ of $G_{m}$ is equal to $Q_{\text {multislice }}^{*^{\prime}}$ of $G_{m}^{\prime}$, i.e. the proposed network size reduction method preserves multislice modularity.

The proof that $Q_{\text {multislice }}^{*^{\prime}}=Q_{\text {multislice }}^{*}$ is as follow: 


$$
\begin{aligned}
Q_{m u l t i s l i c e}^{*^{\prime}}= & \frac{1}{2 \mu^{\prime}} \sum_{m n s r}\left\{\left(w_{m n s}^{\prime}-\gamma_{s} \frac{w_{m s}^{\prime} w_{n s}^{\prime}}{2 w_{s}^{\prime}}\right) \delta_{s r}+C_{m n s r}^{\prime}\right\} \delta\left(g_{m s}, g_{n r}\right) \\
= & \frac{1}{2 \mu^{\prime}} \sum_{s} \sum_{m n}\left(w_{m n s}^{\prime}-\gamma_{s} \frac{w_{m s}^{\prime} w_{n s}^{\prime}}{2 w_{s}^{\prime}}\right) \delta\left(g_{m s}, g_{n s}\right)+ \\
& +\underbrace{\frac{1}{2 \mu^{\prime}} \sum_{s r} \sum_{m n} C_{m n s r}^{\prime} \delta\left(g_{m s}, g_{n r}\right)}_{\text {2nd term }}
\end{aligned}
$$

By applying the same approach followed in [1], it is straightforward to prove that the first term in equation 5 can be rewritten as:

$$
1 \text { st term }=\frac{1}{2 \mu} \sum_{s} \sum_{i j}\left(w_{i j s}-\gamma_{s} \frac{w_{i s} w_{j s}}{2 w_{s}}\right) \delta\left(g_{i s}, g_{j s}\right)
$$

By using equation 4 , it is also easy to show that the second term can be rewritten as:

$$
\begin{aligned}
2 n d \text { term } & =\frac{1}{2 \mu^{\prime}} \sum_{s r} \sum_{m n} C_{m n s r}^{\prime} \delta\left(g_{m s}, g_{n r}\right) \\
& =\frac{1}{2 \mu} \sum_{s r} \sum_{m n}\left(\sum_{i j} C_{i j s r} \delta\left(\operatorname{Com}_{s}(i), m\right) \delta\left(\operatorname{Com}_{r}(j), n\right)\right) \delta\left(g_{m s}, g_{n r}\right) \\
& =\frac{1}{2 \mu} \sum_{s r} \sum_{i j} C_{i j s r} \sum_{m n} \delta\left(\operatorname{Com}_{s}(i), m\right) \delta\left(\operatorname{Com}_{r}(j), n\right) \delta\left(g_{m s}, g_{n r}\right) \\
& =\frac{1}{2 \mu} \sum_{s r} \sum_{i j} C_{i j s r} \delta\left(g_{\operatorname{Com}_{s}(i) s}, g_{\operatorname{Com}_{r}(j) r}\right) \\
& =\frac{1}{2 \mu} \sum_{s r} \sum_{i j} C_{i j s r} \delta\left(g_{i s}, g_{j r}\right)
\end{aligned}
$$

Putting together the first and second terms, we obtain the following:

$$
\begin{aligned}
Q_{\text {multislice }}^{*^{\prime}} & =\underbrace{\frac{1}{2 \mu} \sum_{s} \sum_{i j}\left(w_{i j s}-\gamma_{s} \frac{w_{i s} w_{j s}}{2 w_{s}}\right) \delta\left(g_{i s}, g_{j s}\right)+\frac{1}{2 \mu} \sum_{s r} \sum_{i j} C_{i j s r} \delta\left(g_{i s}, g_{j r}\right)} \underbrace{\text { 2nd term }} \\
& =\frac{1}{2 \mu} \sum_{i j s r}\left\{\left(w_{i j s}-\gamma_{s} \frac{w_{i s} w_{j s}}{2 w_{s}}\right) \delta_{s r}+C_{i j s r}\right\} \delta\left(g_{i s}, g_{j r}\right) \\
& =Q_{\text {multislice }}^{*}
\end{aligned}
$$

In conclusion we proved that nodes belonging to a community in a multislice network can be all replaced by a unique node in the reduced multislice network ( this is a generalization of the work by Arenas et al. in [1]).

\section{An algorithm to discover communities in multislice networks}

To discover communities in multislice networks we propose a greedy algorithm based on a local optimization of the modularity function in equation 2 .

Given a multislice network $G_{m}$, our algorithm consists of two steps repeated iteratively: 


\section{Step 1}

- Initially, we place each node of the network in a different community, so there are as many communities as the nodes in the multislice network (i.e. $\sum_{s} N_{s}$ where $N_{s}$ is the number of nodes in slice $s$ ).

- For each node $i$ in the slice $s$ the gain of $Q_{\text {multislice }}^{*}$ obtained by moving node $i$ in the same community of it's neighbours $j$ is computed. Note that the neighbourhood of a node $i$ is composed by all nodes $i$ is linked to. It also includes those nodes $i$ is linked to through inter-slices coupling.

- Then, node $i$ is placed in the community for which the gain is maximum (and positive).

- Step 1 is performed iteratively until a local maximum of $Q_{\text {multislice }}^{*}$ is reached.

\section{Step 2}

After step 1, we build a new multislice network by applying the size reduction method described in section 3 .

- Each slice of the new network consists of as many nodes as the number of communities found during the step 1 .

- The weight of the intra-slice link between two new nodes $i$ and $j$ is given by the sum of the weights of the links between communities corresponding to nodes $i$ and $j$ respectively (eq. 3). Note that intra-slice links between nodes in the same community are represented by a weighted self-loop link in the corresponding new node.

- The weight $C_{i j s r}$ of the inter-slice links between node $i$ in slice $s$ and node $j$ in slice $r$ is given by the sum of the weights of the links between communities corresponding to nodes $i$ and $j$ placed in slices $s$ and $r$ respectively (eq. 4).

After the second step the number of nodes can diminish drastically, thus speeding up the computation time. To get an idea of how much network size decreases thanks to the proposed reduction method, readers can refer to the work by Arenas et al. [1]. In figure 4 the two steps of our algorithm are graphically illustrated for a network composed by three slices.

In addition, the way the algorithm works permits an implicit discovering of the hierarchical structure of a multislice network. In fact, the network produced at the end of the second step in each pass of the algorithm can be considered as a more higher hierarchical level network. In other words, the hierarchical organization of the network is naturally explored as the algorithm proceeds. In conclusion, our algorithm inherits all the advantages of the Louvain method proposed by V. Blondel et al.[3]:

- It is easy to implement.

- It is very fast (Blondel et al.[3] claim that their implementation is able to find communities in a network of 118 million nodes and 1 billion links in 152 minutes only!)

- It is multi-resolution and naturally gives a hierarchical decomposition of the network.

Additionally, our proposal also allows us to discover communities in multislice networks.

To test our algorithm, we have implemented a prototype written in Python programming language. Since no universally accepted benchmarks for multislice networks currently exist we performed our tests on the examples provided in [14] and specifically we discovered communities in the Zachary Karate network across multiple resolutions. Running our algorithm on this network we obtained the same results reported in [14], thus endorsing the effectiveness of the proposed method. 

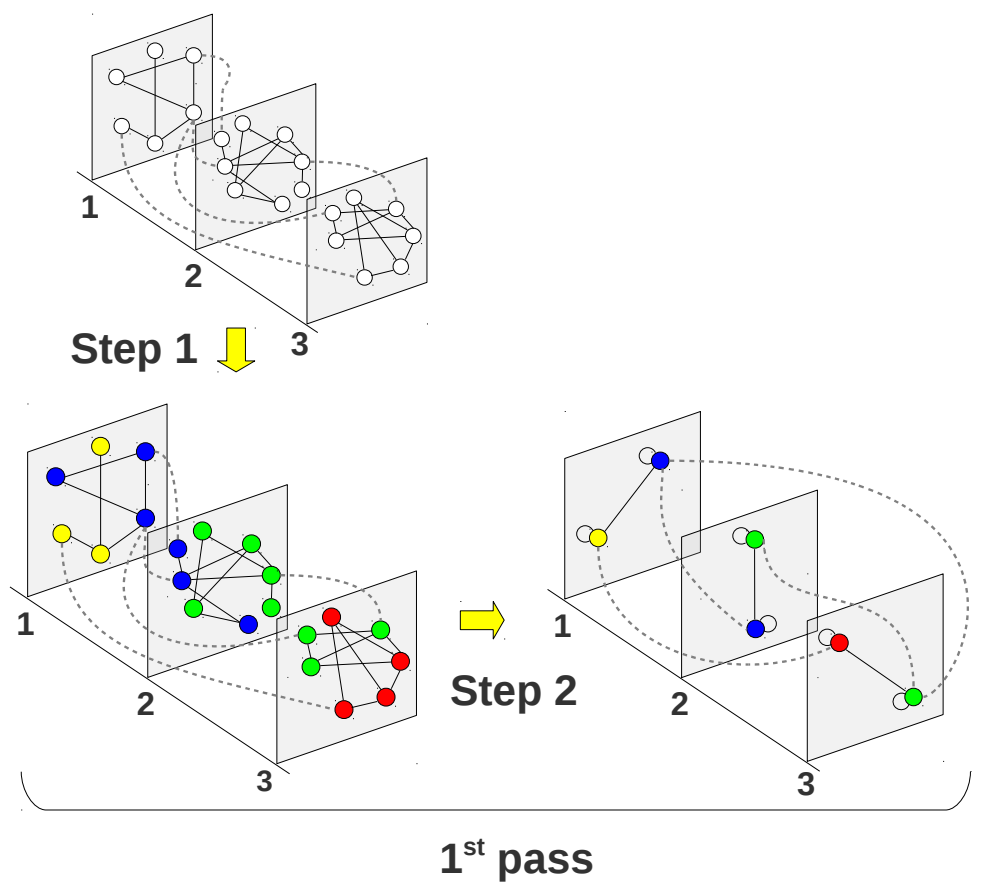

Fig. 2.

\section{Conclusions}

In this paper we presented a greedy algorithm to find communities in multislice networks. Our proposal started from reducing the size of the network without affecting the modularity, so the reduced network partitioning into communities is equivalent to the initial network.

Some issues still have to be addressed, in particular:

- to replace the prototype in Python with an optimized C++ implementation of the greedy algorithm described previously

- to test the proposed approach to real and large networks, in order to assess its effectiveness as well as its performances. This goal is strictly related to the previous one, indeed an optimized algorithm implementation is essential when working on large networks

- to investigate about the definition of (new) benchmarks for multislice networks in addition to currently available benchmarks as [11][12][8]. 


\section{References}

1. A. Arenas, J. Duch, A. Fernandez, and S. Gomez. Size reduction of complex networks preserving modularity. New Journal of Physics, 9:176, 2007.

2. Sitaram Asur, Srinivasan Parthasarathy, and Duygu Ucar. An event-based framework for characterizing the evolutionary behavior of interaction graphs. In Proceedings of the 13th ACM SIGKDD international conference on Knowledge discovery and data mining, KDD '07, pages 913-921, New York, NY, USA, 2007. ACM.

3. Vincent D Blondel, Jean-Loup Guillaume, Renaud Lambiotte, and Etienne Lefebvre. Fast unfolding of communities in large networks. Journal of Statistical Mechanics: Theory and Experiment, 2008(10):P10008, 2008.

4. U. Brandes, D. Delling, M. Gaertler, R. Gorke, M. Hoefer, Z. Nikoloski, and D. Wagner. On modularity clustering. Knowledge and Data Engineering, IEEE Transactions on, 20(2):172-188, Feb. 2008.

5. Deepayan Chakrabarti, Ravi Kumar, and Andrew Tomkins. Evolutionary clustering. In Proceedings of the 12th ACM SIGKDD international conference on Knowledge discovery and data mining, KDD '06, pages 554-560, New York, NY, USA, 2006. ACM.

6. D. J. Fenn, M. A. Porter, M. McDonald, S. Williams, N. F. Johnson, and N. S. Jones. Dynamic communities in multichannel data: An application to the foreign exchange market during the 2007-2008 credit crisis. Chaos, 19(3):033119-+, September 2009.

7. Santo Fortunato. Community detection in graphs. Physics Reports, 486:75-174, 2010.

8. M. Girvan and M. E. J. Newman. Community structure in social and biological networks. Proceedings of the National Academy of Sciences of the United States of America, 99(12):7821-7826, June 2002.

9. John Hopcroft, Omar Khan, Brian Kulis, and Bart Selman. Tracking evolving communities in large linked networks. Proceedings of the National Academy of Sciences, 101:5249-5253, April.

10. Ravi Kumar, Jasmine Novak, and Andrew Tomkins. Structure and evolution of online social networks. In Proceedings of the 12th ACM SIGKDD international conference on Knowledge discovery and data mining, KDD '06, pages 611-617, New York, NY, USA, 2006. ACM.

11. Andrea Lancichinetti and Santo Fortunato. Benchmarks for testing community detection algorithms on directed and weighted graphs with overlapping communities. Phys. Rev. E, 80(1):016118, Jul 2009.

12. Andrea Lancichinetti, Santo Fortunato, and Filippo Radicchi. Benchmark graphs for testing community detection algorithms. Phys. Rev. E, 78(4):046110, October 2008.

13. Jure Leskovec, Kevin J. Lang, Anirban Dasgupta, and Michael W. Mahoney. Community structure in large networks: Natural cluster sizes and the absence of large well-defined clusters. Internet Mathematics, 6(1):29-123, 2009.

14. Peter J. Mucha, Thomas Richardson, Kevin Macon, Mason A. Porter, and Jukka-Pekka Onnela. Community structure in time-dependent, multiscale, and multiplex networks. Science, 328(5980):876-878, May 2010.

15. M. E. J. Newman and M. Girvan. Finding and evaluating community structure in networks. Physical Review E, 69:026113, 2004.

16. Gergely Palla, Albert lszl Barabsi, Tams Vicsek, and Budapest Hungary. Quantifying social group evolution. Nature, 446:2007, 2007. 